\title{
Automated classification of tailed bacteriophages according to their neck organization
}

\author{
Anne Lopes ${ }^{1,2,3}$, Paulo Tavares ${ }^{4}$, Marie-Agnès Petit ${ }^{5,6}$, Raphaël Guérois ${ }^{1,2^{*}+}$ and Sophie Zinn-Justin ${ }^{1,2^{*}+}$
}

\begin{abstract}
Background: The genetic diversity observed among bacteriophages remains a major obstacle for the identification of homologs and the comparison of their functional modules. In the structural module, although several classes of homologous proteins contributing to the head and tail structure can be detected, proteins of the head-to-tail connection (or neck) are generally more divergent. Yet, molecular analyses of a few tailed phages belonging to different morphological classes suggested that only a limited number of structural solutions are used in order to produce a functional virion. To challenge this hypothesis and analyze proteins diversity at the virion neck, we developed a specific computational strategy to cope with sequence divergence in phage proteins. We searched for homologs of a set of proteins encoded in the structural module using a phage learning database.

Results: We show that using a combination of iterative profile-profile comparison and gene context analyses, we can identify a set of head, neck and tail proteins in most tailed bacteriophages of our database. Classification of phages based on neck protein sequences delineates 4 Types corresponding to known morphological subfamilies. Further analysis of the most abundant Type 1 yields 10 Clusters characterized by consistent sets of head, neck and tail proteins. We developed Virfam, a webserver that automatically identifies proteins of the phage head-neck-tail module and assign phages to the most closely related cluster of phages. This server was tested against 624 new phages from the NCBI database. $93 \%$ of the tailed and unclassified phages could be assigned to our head-neck-tail based categories, thus highlighting the large representativeness of the identified virion architectures. Types and Clusters delineate consistent subgroups of Caudovirales, which correlate with several virion properties.

Conclusions: Our method and webserver have the capacity to automatically classify most tailed phages, detect their structural module, assign a function to a set of their head, neck and tail genes, provide their morphologic subtype and localize these phages within a "head-neck-tail" based classification. It should enable analysis of large sets of phage genomes. In particular, it should contribute to the classification of the abundant unknown viruses found on assembled contigs of metagenomic samples.
\end{abstract}

Keywords: Bacteriophage, Profile-profile comparison, Virion, Gene organization, Evolution

\section{Background}

Bacteriophages, which are defined as viruses that infect and replicate within bacteria, constitute the largest known group of viruses [1,2]. They occur everywhere in the biosphere where bacteria are found, their habitats being as diverse as oceans, topsoils, plants or animals. The total number of phage species is estimated to reach millions $[3,4]$, and their classification remains a challenge nowadays. Whereas molecular biology has permitted for most

\footnotetext{
* Correspondence: raphael.guerois@cea.fr; sophie.zinn@cea.fr

${ }^{\dagger}$ Equal contributors

${ }^{1}$ CEA, iBiTecS, Gif-sur-Yvette, F-91191 Paris, France

Full list of author information is available at the end of the article
}

living organisms a progressive shift from character-based classifications to classifications based on genetic markers [5], no satisfying method for classifying phages exists at present. Phages were initially sorted according to two characters: the nature of their encapsidated nucleic acid and their virion morphology. More than $96 \%$ of them are tailed phages: they constitute the Caudovirales order [6]. They encapsidate double stranded DNA genomes. Moreover, their viral particle is formed by a head, mainly constituted by an icosahedral capsid that protects the viral genome, and a tail specialized in DNA delivery inside the bacterial host. Caudovirales are divided into Siphoviridae, Myoviridae and Podoviridae families depending on the nature 
of their tail, which is respectively long and non-contractile, long and contractile, or short. Phenotypic observation does not permit finer grained structural distinction among these tailed phages, so that molecular tools are needed to further classify them [7]. At this family level, some classification problems arise, as some phages can be separated into Sipho-, Myo- and Podoviridae, while having closely related genomes $[8,9]$.

Phage hierarchical trees were obtained from the analysis of capsid proteins [10]. Terminase [11] and portal [12] proteins were further described as potential markers of phage diversity, as well as tape measure proteins in long-tail bacteriophages [13]. However, the selected gene is sometimes not detected in some of the phages, thus excluding these phages from the classification $[12,13]$ and limiting the use of the gene as a marker for biodiversity studies [8]. Moreover, a single gene does not provide a global view of the virion architecture, thus hindering phage classification as a function of a general virion structural organisation.

Phage hierarchical trees were recalculated based on whole genome analyses $[9,14]$. To some extent this approach permitted the definition of some genera and subfamilies among Myo- and Podoviridae, but it seemed unsuccessful with respect to Siphoviridae. Decisive in such approaches is the capacity to group together large enough sets of orthologs, which remains difficult given the remarkable level of sequence divergence between phage proteins. Not only do phage genomes mutate more rapidly than bacterial genomes, but they also recombine more readily, so that the notions of phage species and hierarchical classification can be questioned. Using a similar estimate of relatedness based on the amount of shared genes, but departing from the hierarchical classification, an attempt at representing phage relatedness with graph theory revealed a densely connected network of all Caudovirales [15]. However, it is difficult to extract from such an analysis the relationship between phage classification and specific phage properties as for example virion morphology. In order to understand how highly divergent phages encode for a virion capable of infecting bacteria, we focused the analysis on a set of genes belonging to the "structural" module involved in virion assembly and host infection.

Despite phage divergent and complex evolution, the thorough molecular analysis of a few paradigmatic tailed phages belonging to different morphological classes, such as Siphoviridae SPP1 and $\lambda$, Myoviridae T4 and Podoviridae P22 and $\Phi 29$, suggested that only a limited number of structural solutions are used in order to produce a functional virion [16-19]. To challenge this hypothesis, we searched for homologs of a set of virion proteins functionally characterized through the study of the assembly pathway of the corresponding phages (Figure 1, Table 1 and Experimental procedures). Protein names sometimes differ for the various model phages that were studied, and are unified in Figure 1 for the sake of clarity. Proteins from the head (Major Capsid Protein or MCP, portal and terminase) and the tail (Major Tail Protein or MTP, sheath) of bacteriophages are generally well conserved, and could be detected with standard bioinformatics strategies. In contrast, proteins lying at the interface between the head and tail components, the so-called Ad, Hc and Tc head-to-tail connection proteins (see Figure 1 for definitions), can be much more difficult to detect due to drastic sequence divergence.

To obtain a global view of the "structural module" in bacteriophages and probe whether newly sequenced phages can be assigned to already known systems, we developed a specific computational strategy able to cope with the high divergence and plasticity of phage genomes. A sensitivity-enhanced bioinformatics approach based on profile-profile comparisons was initially used [29]. We further improved this method by performing systematic gene context analyses and successfully detected the head-to-tail connection proteins in $91 \%$ of 328 genomes of tailed and unclassified phages. Based on the occurrence of these proteins, phages were classified into 4 Types and their relationships with known morphological subfamilies (Siphoviridae, Myoviridae, and Podoviridae) were defined. Next, a similarity metric between phages, combining profile-profile comparison scores with sequence identities, was developed to provide a finer classification of virion architectures within every Type. For the latter step, not only proteins from the head-to-tail connection (neck) were considered but also components of the head and the tail. As a result, the most abundant Type 1 could be divided into 10 Clusters, some exclusively containing Siphoviridae or Myoviridae, and others aggregating both Sipho- and Myoviridae. We developed a Webserver, called Virfam, to map any novel phage genome on our classification scheme. By testing this server on 624 phage genomes from the NCBI database not yet included in our dataset, we confirmed the large applicability of our classification approach, as $93 \%$ of them could be classified within one of the Type/Cluster classes. Beyond phage classification, this study also provides insights into the possible organization of ancestral neck modules in Caudovirales.

\section{Implementation}

\section{HMM Profiles generation for proteins of the learning} database

For all the 28300 phage sequences contained in the Aclame database (version 0.4) [30], a profile was built following the protocol described in [29]. In the latest release of Aclame (version 0.4), 465 bacterial phage genomes are accessible but only 447 fulfilled the criteria of being bacteriophages (10 are phages infecting Archeae) 


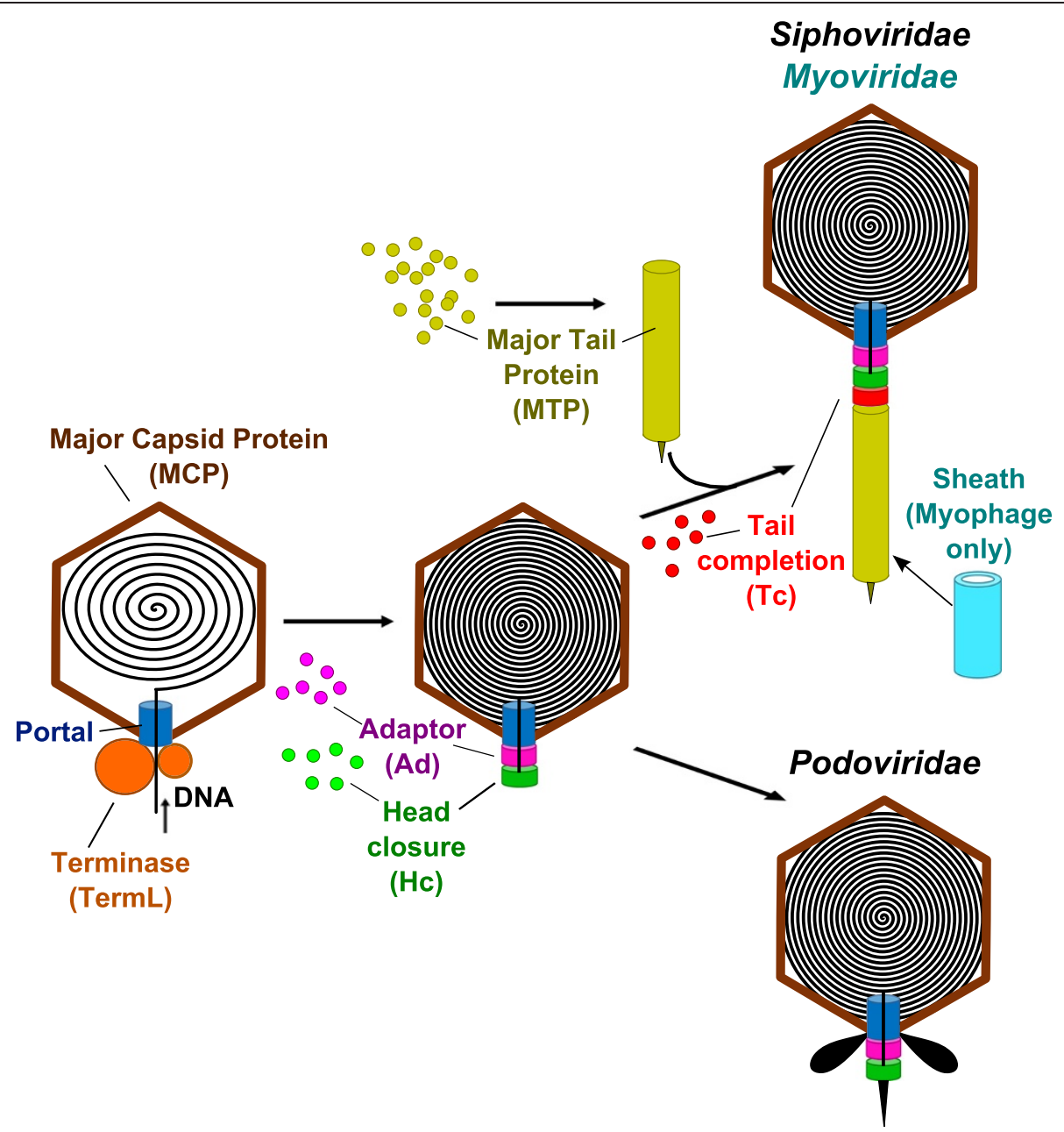

Figure 1 Assembly pathway of tailed bacteriophages. In the tailed phages, capsid assembly starts with the construction of an icosahedral protein lattice called procapsid, essentially composed of a major capsid protein (noted MCP in brown in Figure 1). At a specialized vertex of the procapsid, the dodecameric portal protein (Portal in blue) forms a channel which is the docking point for an ATPase complex called terminase. This complex normally contains multiple copies of a large subunit with ATPase and endonuclease activities (TermL in orange), and a small DNA binding subunit that recognizes the cognate viral DNA Sun et al. [20]. It translocates viral dsDNA into the procapsid cavity through the portal channel. When DNA packaging is completed, the terminase motor disassembles and the portal dodecamer recruits head-completion proteins to prevent leakage of the viral DNA. One such protein directly binds to the portal: it is called the adaptor protein (Ad in magenta); it can also be supplemented with the so-called head-closure protein ( $\mathrm{Hc}$ in green) [18,21-23]. Altogether the head-completion proteins provide a platform for completion of short tail assembly in Podoviridae [24,25] as well as for docking of pre-assembled long tails in Sipho- and Myoviridae [26-28]. Located at one end of the Sipho- and Myoviridae long tails, the tail-completion protein (Tc in red) allows for the tail attachment to the head. Head- and tail-completion proteins form the head-to-tail connection and, together with the portal protein, constitute the virion's neck. The major tail protein (MTP in kaki) is the main component of the tail tube structure. In Myoviridae, the surrounding tail sheath protein (Sheath in cyan) contracts upon host infection, initiating viral DNA injection in the host cell.

with treatable sequences ( 8 were not properly implemented in Aclame). Briefly, for every sequence, a 3 iteration PSI-Blast [31] search was performed against the non-redundant (nr70 (dec. 2010)) database using a cutoff e-value of $10^{-4}$. Whenever an iteration retrieved more than 1000 homologs, the previous iteration was kept so as to prevent divergence issues. The resulting multiple sequence alignment was filtered so as to keep the 100 most diverse sequences and was converted into a Hidden-Markov Model profile using the HHsuite programs [32] integrating the secondary structure prediction from Psi-pred [33]. The detailed bioinformatics procedure described below was followed for each of the different component of the head-neck-tail module. To evaluate the sensitivity gains provided by profile-profile comparisons (HHsuite) with respect to profile-sequence ones (PSI-Blast), as presented in Table 2, we followed the procedure described in Additional file 1: Method S1. 
Table 1 Functionally characterized head- and tail-completion proteins of tailed bacteriophages classified in Aclame

\begin{tabular}{|c|c|c|c|c|c|}
\hline Phage & Order & Head-completion Ad & Head-completion Hc & Tail-completion Tc & Neck type \\
\hline SPP1 & Siphoviridae & $\mathrm{gp} 15^{1}$ & $\mathrm{gp} 16^{2}$ & $g p 17^{3}$ & 1 \\
\hline Lambda & Siphoviridae & gpW & $\mathrm{gpFII}^{2}$ & $\mathrm{gpU}^{3}$ & 1 \\
\hline HK97 & Siphoviridae & gp6 $^{1}$ & gp7 & gp9* & 1 \\
\hline T4 & Myoviridae & gp13 & gp14 & gp1 $15^{3}$ & 2 \\
\hline P22 & Podoviridae & gp $4^{1}$ & gp10 & - & 3 \\
\hline Ф29 & Podoviridae & gp11 & - & - & 4 \\
\hline
\end{tabular}

*Refers to a protein identified through this study.

Head- and tail-completion proteins extracted from the literature are listed in this table. Proteins of known 3D structure are indicated in bold. Structural homologies between proteins that were experimentally demonstrated are indicated by similar numbers in superscript. Neck types result from the current analysis. In particular, SPP1, $\lambda$ and HK97 are gathered within the same phage type because they are predicted as exhibiting homologous head- and tail completion proteins. GpW of Siphoviridae phage $\lambda$ corresponds to a rare alternative playing the role of SPP1 gp15 at the head-to-tail connection (although gpW and gp15 share no homology at the sequence and structural levels).

\section{Detection of the head and tail proteins within the learning database}

We first searched for the major capsid proteins, portal proteins, terminases, major tail proteins and Sheath proteins within Aclame 0.4. The various components of the head and the tail were identified starting from proteins whose 3D structures were solved and using our iterative profile-profile comparison procedure with a probability confidence threshold of $90 \%$ as described in [29]. In the case of the major capsid proteins, the 3D structures are known from X-ray data for the Siphophage HK97 and the Myophage T4. The HK97 and T4 major capsid proteins exhibit a similar polypeptide fold [34]. Consistently, they were detected as related by HHsearch with a confidence score of $93 \%$. We used their profiles as starting points in order to detect other proteins belonging to the major capsid superfamily. 290 major capsid proteins were identified, including the capsid proteins of the
Siphophages SPP1, $\lambda, \Phi \varepsilon 125$, the Myophages P2, Mu and the Podophages T3, T7, $\varepsilon 15$, P22, PZA. For several of these phages, a high resolution EM structure of the capsid is available, and the EM data are consistent with the existence of a common major capsid protein fold [35-38]. In the case of the portal proteins, the $3 \mathrm{D}$ structures are known for phages $\Phi 29$, SPP1 and P22. The portal proteins of these phages exhibit a common fold [39,40]. We used their profiles as starting seeds and identified 308 portal proteins, including those of phages HK97, $\lambda, \Phi \varepsilon 125, \mathrm{~T} 4$, P2, Mu, T3, T7 and $\varepsilon 15$. 3D structures of terminase large subunits are known for phages T4 (whole gp17 protein [41] and nuclease domain [42]) and SPP1 (nuclease domain [43]). Here again, despite a very low sequence identity, the fold of the nuclease domain is conserved. Using the profiles of the SPP1 and T4 terminases as starting point, 307 large terminases were identified, including those of phages $\mathrm{HK} 97, \lambda, \Phi \varepsilon 125, \mathrm{P} 2, \mathrm{Mu}, \mathrm{T} 3$,

Table 2 Detected head- and tail-completion proteins of Aclame

\begin{tabular}{|c|c|c|c|c|c|c|}
\hline & $\begin{array}{l}\text { Detected with } \\
\text { Psi-Blast }\end{array}$ & $\begin{array}{l}\text { Detected with } \\
\text { HHsearch at } 90 \%\end{array}$ & $\begin{array}{l}\text { Detected only at } 70 \% \text { using } \\
\text { a combined approach }\end{array}$ & $\begin{array}{l}\text { Percentage of proteins detected } \\
\text { with the combined approach }\end{array}$ & Exceptions & Total protein number \\
\hline Ad1 & 86 & $191 *$ & 10 & 5 & 12 & $213 *$ \\
\hline $\mathrm{Hc} 1$ & 71 & 158 & 4 & 2 & 5 & 167 \\
\hline TC1 & 41 & 166 & 27 & 13 & 14 & 207 \\
\hline $\mathrm{Ad} 2$ & 17 & 17 & 0 & 0 & 0 & 17 \\
\hline $\mathrm{Hc} 2$ & 16 & 16 & 0 & 0 & 0 & 16 \\
\hline Tc2 & 16 & 16 & 0 & 0 & 0 & 16 \\
\hline Ad3 & 8 & 8 & 30 & 60 & 12 & 50 \\
\hline $\mathrm{HC3}$ & 17 & 51 & 0 & 0 & 0 & 51 \\
\hline Ad4 & 10 & 10 & 0 & 0 & 0 & 10 \\
\hline Total & 282 & 633 & 71 & 10 & 43 & 747 \\
\hline
\end{tabular}

*Including 5 gpW-like Ad1.

Head- and tail-completion proteins detected using either the profile-sequence comparison tool PSI-BLAST, the profile-profile comparison tool HHsearch with a threshold of $90 \%$ or an approach combining the HHsearch tool at a threshold of $70 \%$ with analysis of the gene contexts are detailed (percentages correspond to HHsearch confidence thresholds). Proteins counted in the "Exceptions" column correspond to proteins that were manually detected, either because their HMM profile was built from very few sequences (hindering profile-profile comparisons) but they showed consistent secondary structure prediction and genome localisation, or because they were detected by HHsearch but their genome positioning slightly deviated from the canonical positioning. The Aclame references of the detected neck proteins, as well as of the identified head and tail proteins, are displayed in Additional file 2: Table S1. 
T7, P22 and $\varepsilon 15$. We did not find the terminase of $\Phi 29$, consistent with a previous bioinformatics study that clearly demonstrated the existence of two structurally distinct families of large terminases, one of these families being found in most tailed bacteriophages, and the other family being only found in $\Phi 29$-like phages [44]. In the case of the major tail proteins, only one $3 \mathrm{D}$ structure is available, that of gpV from phage $\lambda$ [19], from which we detected 185 tail proteins, including those of the Siphophage SPP1 and the Myophage P2. However, we did not identify major tail proteins for T4-like Myophages. Thus, we also searched homologs for the well-characterized T4 major tail protein gp19 and found 16 additional homologous tail proteins. Search for the fold of these proteins using HHpred [45] against a profile database derived from the Protein Data Bank revealed that T4-like MTP likely share the fold of phage $\lambda$ major tail protein gpV (probability score higher than 90\%). This suggests that myophages of the T4 family also present a tail protein evolutionary linked to that of the siphophages. Finally, regarding Sheath proteins, the 3D structures are known for phages T4 [46] and PhiKZ [47]. These structures exhibit a common structural core [47] that was not detected by HHsearch. 70 Sheath proteins were identified starting from the T4 protein, out of the 74 Myophages in Aclame. Only 2 Sheath proteins were identified starting from the PhiKZ protein, corresponding to the giant phages PhiKZ and PhiEL. Within the 2 myophages left without any assigned Sheath protein, C-st was experimentally shown to be a myophage and should have a Sheath protein. On the opposite, P4 is a satellite bacteriophage coding for 14 proteins, which consistently lacks a Sheath protein. At this stage, we identified head, neck and tail proteins within most of the 447 phages of Aclame.

\section{Detection of the head- and tail-completion proteins using remote homology search and genetic context}

A bioinformatics procedure was designed in order to systematically detect the phage head-completion and tail-completion proteins, also called head-to-tail connection proteins, within the Aclame database (Figure 2A). To do so, the characterized head-to-tail connection proteins of phages SPP1, $\lambda$, HK97, T4, P22 and Ф29 were taken as starting point. Next, their profiles were compared to the database of $28300 \mathrm{HMMs}$ profiles using the profile-profile comparison algorithm HHsearch [32].

\section{Type 1}

First, all possible head-to-tail connection proteins Ad1, Hc1 and Tc1 were identified among the 447 genomes by searching for homologs of the SPP1 gp15, gp16 and gp17 proteins using the program HHsearch with a high confidence probability threshold of $90 \%$. Next, the intergene distances between the detected Ad1, Hc1 and Tc1 proteins were calculated and averaged on all genomes.
The resulting mean inter-gene distances were 1, 2 and 3 for the (Ad1, Hc1), (Hc1, Tc1) and (Ad1, Tc1) couples, respectively, corresponding to the gene ordering: Ad1Hc1-x-Tc1. They could be organised into a mean distance matrix characteristic of Type 1 neck gene organisation. We also calculated for each couple the standard deviation on the inter-gene distances. From these calculations we deduced a tolerated distance matrix, formed by adding to each mean distance matrix term twice the inter-gene distance standard deviation. The tolerated distance matrix terms were 2, 4 and 5 for the (Ad1, Hc1), (Hc1, Tc1) and (Ad1, Tc1) couples, respectively. Finally, we repeated the iterative profile-profile search with $\mathrm{HHsearch}$, relaxing the probability threshold from $90 \%$ to $70 \%$, and keeping only the detected proteins whose gene position was consistent with the tolerated matrix distance. As a result, more than 500 neck proteins were recovered (Table 2).

To control that no false positives were recovered by our relaxed conditions, we checked that we never detected more than one Ad1, one Hc1 or one Tc1 per phage. We also verified our results in the light of well characterized phages. Tc1 being a tail completion protein involved in long tail assembly [26,48], we anticipated that a neck containing Tc1 would not be found in Podoviridae phages. Consistently, no neck containing Tc1 proteins could be detected in Podoviridae phages. Some phages do not encode the whole Ad1, Hc1, Tc1 panoply. For instance, the neck of phage $\lambda$ is made of an Hc1 (called gpFII), a Tc1 (called gpU) but no Ad1. Instead, the gpW protein, with no sequence or structural similarity to Ad1, is required for stabilization of the DNA within the head and for addition of the Hc1 protein. This typical case of functional replacement suggests that $\mathrm{GpW}$ is positioned in the virion between the portal and the Hc1 proteins [49]. In our study, a small group of 10 Siphoviridae and Myoviridae phages exhibit Hc1 and Tc1 proteins but no Ad1 proteins. Within these phages, 4 Siphoviridae (in which phage $\lambda$ ) and 1 Myoviridae encode a protein similar to gpW using a confidence threshold of $95 \%$ and systematically located at distances of 6 to 7 from Hc1 and 9 from Tc1. Thus, our procedure did not detect any spurious remote homologs in the particular cases of necks comprising a gpW-like protein.

We specifically analyzed the genomes lacking only one of the Ad1, Hc1 and Tc1 elements. Searching within the limits imposed by the tolerated distance matrix, 3 Ad1, 5 Hc1 and 13 Tc1 proteins were identified in Siphoviridae. These proteins were predicted as having the same secondary structure patterns as the missing ones and were located at distances from the other identified neck proteins compatible with the tolerated distance matrix. They were not automatically selected by our initial profileprofile procedure because their HMM profile was built from a too limited number of sequences (less than 5 


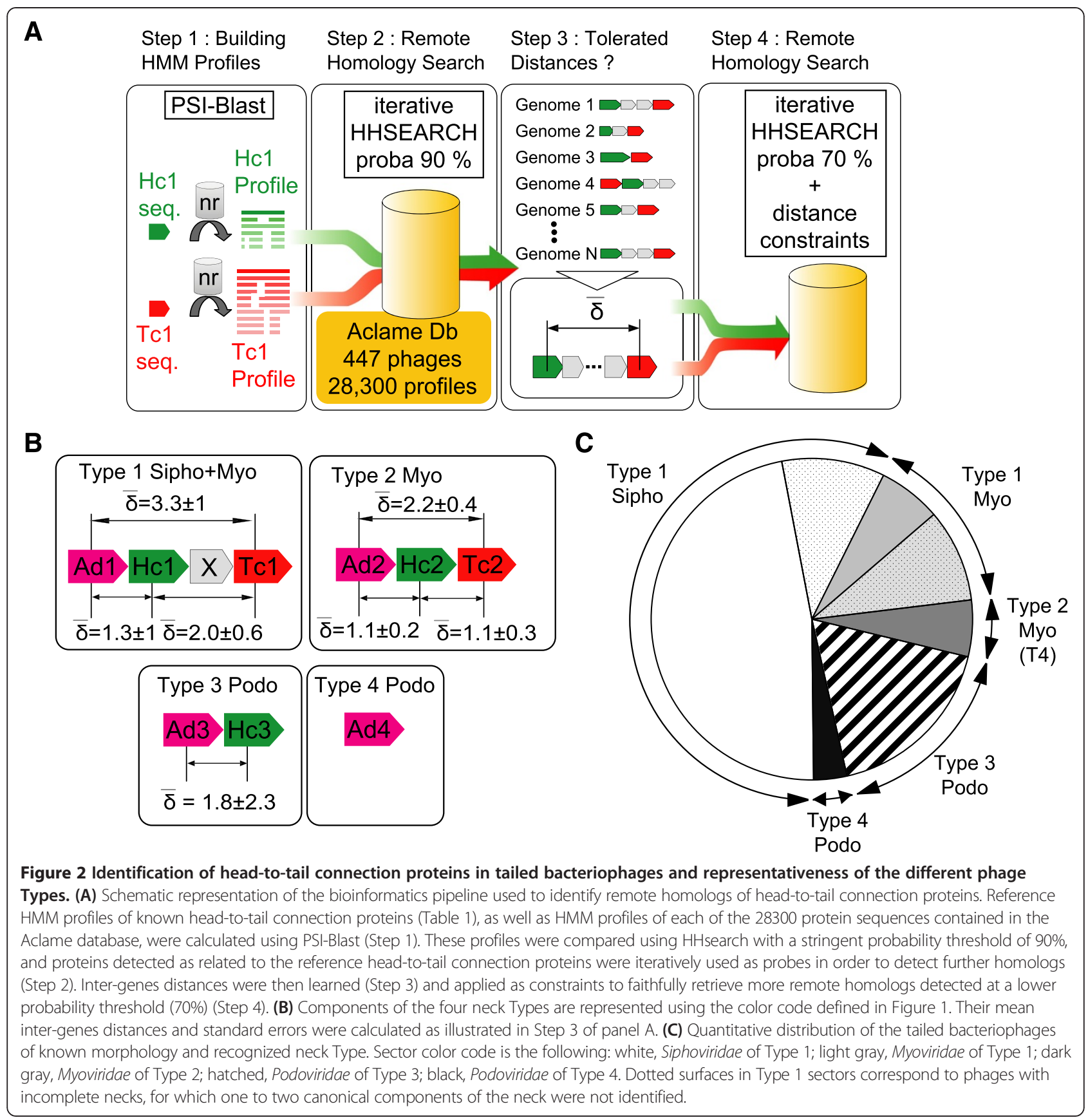

homologous sequences in the $\mathrm{nr}$ database retrieved at the PSI-Blast stage). Similarly, we identified 1 Ad1 and 1 Tc1 protein in Myoviridae phages exhibiting less than 4 homologous sequences in their HMM profile. Finally, 8 additional Ad1 were identified in Myoviridae phages exhibiting a Tc1 protein, which are separated by 6 to 8 proteins from Tc1 (the tolerated matrix imposed a maximal value of 5 ). In the latter case, the $8 \mathrm{Ad} 1$ belong to an Aclame family also comprising 6 other proteins previously identified as Ad1. All these neck proteins are described in Table 2 and Additional file 2: Table S1.

\section{Type 2}

The head-to-tail connection proteins Ad2, Hc2 and Tc2 were identified by searching for homologs of the T4 gp13, gp14 and gp15 proteins using the program HHsearch with a $90 \%$ confidence threshold. Inter-gene distances were calculated between the identified proteins resulting in mean distances of 1,1 and 2 for the (Ad2, Hc2), (Hc2, Tc2) and (Ad2, Tc2) couples, respectively. This corresponds to the ordering: Ad2-Hc2-Tc2. The tolerated distance was calculated as above by summing the mean and twice the standard deviation inter-gene distances. The tolerated 
distances were 2, 2 and 3 for the (Ad2, Hc2), (Hc2, Tc2) and (Ad2, Tc2) couples, respectively. As for Type 1 , we constrained the search using this inter-gene tolerated distance matrix and repeated the iterative profile-profile search with $\mathrm{HHsearch}$, relaxing the probability threshold from $90 \%$ to $70 \%$. As a result, we identified 49 Type 2 neck proteins (Table 2; Additional file 2: Table S1).

\section{Type 3}

The head-to-tail connection proteins Ad3 and Hc3 were identified by searching for homologs of the P22 gp4 and gp10 proteins with HHsearch and a 90\% confidence threshold. A tolerated inter-gene distance of 2 was calculated and used to constrain the search while relaxing the probability threshold of HHsearch at $70 \% .89$ additional Type 3 neck proteins were thus detected.

We searched for missing Ad3 and Hc3 proteins using the secondary structure predictions and the knowledge of their relative positions in the phage genomes. We identified 1 additional Ad3 protein with consistent predicted secondary structure pattern and inter-gene distance to its Hc3 neighbour. Here again lack of homologous sequences in the original profile hindered the homology detection by the HHsearch algorithm. Moreover, in 3 additional Podoviridae, Ad3 and Hc3 were identified but their relative distance was comprised between 3 and 6 . Finally, in 8 additional phages (4 Podoviridae and 4 unclassified phages), 2 Ad3 and 1 Hc3 were identified per phage, the 2 Ad3 having a relative distance of 1 to 2 and Hc3 having a relative distance to Ad3 of 4 to 15 . All these neck proteins are described in Table 2 and Additional file 2: Table S1.

\section{Type 4}

For this last type, Ad4 proteins were searched using the $\Phi 29$ gp11 protein as a seed for the iterative profile-profile procedure. Ten Ad4 proteins were detected, that all belong to Podoviridae (Table 2).

\section{Scoring of the evolutionary divergence between head-neck-tail modules for phage classification}

Our analysis identified a set of 9 classes of proteins of the head-neck-tail module comprising 2 classes from the head (major capsid, terminase), 5 from the neck (Portal, Ad, Hc, Tc, Ne) and 2 from the tail (major tail, Sheath). Every class encompasses a number of Aclame protein families that were previously thought unrelated and which are now connected through remote homology relationships by the procedure described above. Taking advantage of this global view of the head-neck-tail organisation, we searched for a metric which would account for the evolutionary divergence between the phage components of the head-neck-tail module. We used a score depending both on the HHsearch probability (ProbaHHsearch) but also on the sequence identity (PercentIdentity) for closely related sequences.

$$
\begin{aligned}
\text { SimScore }= & 1 * \text { ProbaHHsearch } \\
& +0.1 * \text { PercentIdentity }
\end{aligned}
$$

The weight of 0.1 assigned to the PercentIdentity factor ensured that the latter term only contributes when ProbaHHsearch caps at $100 \%$ because values of ProbaHHsearch below 100\% generally correspond to remote homology relationships with PercentIdentity below 25\%. For every pair of phages and for all the components of the capsid-neck-tail module (out of the nine possible components), a similarity score was calculated using equation 1 . The mean value of all these scores was computed over all the components of the module common to both phages (Portal, Ad, Hc etc....) in order to yield the similarity score between the pair of phages. It was calculated by adding scores corresponding to superfamilies detected in both phages and ignored superfamilies only detected in one of them.

The mean value was used as a metric of similarity of the capsid-neck-tail module between two phages and a $\mathrm{NxN}$ matrix of averaged scores was built from the systematic cross-comparison of all $\mathrm{N}$ phages of each type. To group together phages sharing similar average scores, the $\mathrm{NxN}$ matrices were then clustered using a hierarchical agglomerative clustering with WPGMA method (Weighted Pair Group Method with Averaging) together with Euclidian distance and a tree corresponding to each neck type was built from these calculations using the ete2 library [50].

For the phage classification step, we took into account all phages with at least two detected head-to-tail connection proteins in the case of Type 1 and 2 phages (including T5 which has only Ad1 and Tc1 proteins), and one detected protein in the case of Type 3 and 4 phages (including N4 which has only one Ad3 protein). In the Webserver, in order to increase sensitivity, all head-to-tail connection proteins detected with a HHsearch threshold higher than $70 \%$ are displayed.

\section{Automatic identification of neck proteins and classification of necks in tailed bacteriophages through the Virfam server}

To enlarge our study to newly sequenced phages, we built the Virfam webserver interface through which users can (1) detect the proteins involved in the neck structure of their phage of interest, (2) identify the type of neck, and deduce the likely morphological family of the phage, (3) display the organisation of the genes encoding the major capsid protein, large terminase, portal, neck, major tail and sheath proteins and (4) locate the given phage 
within the classification built in this work. After submission through this interface, each input protein is aligned using an HHsearch-based protocol against the Virfam collection of 28000 profiles generated for the present work. Phage neck proteins are thus automatically identified and the phage neck architecture is described.

\section{Results}

Detection of head and tail proteins within the learning dataset

Our structural classification of phages relies on the detection of 9 genes coding for head, neck and tail proteins. We first searched for homologs of 5 head and tail proteins using the homology detection tool HHsearch [32], which relies on the comparison between pairs of multiple sequence alignments (also called profiles). This tool can reveal more remote homologies between proteins than the profile-sequence comparison tool PSI-Blast [31]. It was used with a high confidence probability threshold of $90 \%$ to search for the portal, large terminase subunit (noted here TermL but also often abbreviated as TerL) and major capsid protein (MCP) on the head side, the major tail (MTP) and sheath proteins on the tail side, among a set of 447 complete phage genomes, including 303 tailed phages (Caudovirales), 119 non tailed phages and 25 uncharacterised phages (taken from Aclame version 0.4; see Virfam server for details). The 5 head and tail proteins could be detected in most tailed phages. Details about the initial sequences used for the search, corresponding to proteins of known 3D structures, are given in the Experimental procedure section and the complete list of detected proteins can be found in Additional file 2: Table S1.

\section{Detection and classification of head- and tail-completion} proteins within the learning dataset

Once the head and tail proteins were identified, we focused on the head-to-tail connection proteins to capture the diversity of neck organisations in tailed phages. We concentrated on the identification of a maximum of highly divergent head-to-tail connection proteins. Therefore, we identified from the literature several proteins characterized as head-and tail- completion proteins in a number of model phages (Table 1), and we search for homologs of these proteins within the genomes of the same 447 completely sequenced phages. Our homology search was performed using the homology detection tool HHsearch, which was now used iteratively to detect highly remote homologs (Figure 2A). A stringent confidence probability threshold of $90 \%$ was again chosen to identify homologs of our list of starting proteins (Table 1) among the 28300 proteins encoded by the 447 phages. We detected 633 phage head- and tail-completion proteins (compared to only 282 when using PSI-Blast, see Table 2). Most of them were highly divergent from one another (pairwise identity in the $10-20 \%$ range). However, despite this drastic divergence, the 633 detected proteins combined into a limited number of 4 major neck architectures, or neck "Types" (Tables 1 and 3).

These neck Types display different complexities: they involve one to three head-to-tail connection proteins (a fourth protein called Ne1 will be taken into account later on). Type 1 necks (or SPP1-like) are found in Siphoviridae and Myoviridae. They adopt a structural organisation similar to that of the Siphoviridae phage SPP1 and comprise three head-to-tail connection proteins: two headcompletion proteins homologous to SPP1 gp15 (Adaptor, noted Ad1) and SPP1 gp16 (Head closure, noted Hc1) and a tail-completion protein homologous to SPP1 gp17 (noted Tc1). Exception to this rule is found for 5 phages (among 219 Type 1 neck phages), which exhibit an adaptor homologous to gpW of phage $\lambda$, a protein with a structure distinct from that of SPP1 gp15 [51]. For these 5 phages, the difference is limited to the adaptor since bona fide homologs of Hc1 and Tc1 are detected. Type 2 necks (T4-like)

Table 3 Detected neck modules in the phages classified in Aclame

\begin{tabular}{|c|c|c|c|c|c|c|c|}
\hline & $\begin{array}{c}\text { Type } 1 \text { with } 4 \\
\text { head-to-tail } \\
\text { connection } \\
\text { proteins (Ad1, Hc1, } \\
\text { Tc1, Ne1) }\end{array}$ & $\begin{array}{c}\text { Type } 1 \text { with } 2 \text { or } \\
3 \text { head-to-tail } \\
\text { connection } \\
\text { proteins (and only } \\
1 \text { in brackets) }\end{array}$ & $\begin{array}{c}\text { Type } 2 \text { with } 3 \\
\text { head-to-tail } \\
\text { connection } \\
\text { proteins (and only } \\
1 \text { in brackets) }\end{array}$ & $\begin{array}{c}\text { Type } 3 \text { with } 2 \\
\text { head-to-tail } \\
\text { connection } \\
\text { proteins (and only } \\
1 \text { in brackets) }\end{array}$ & $\begin{array}{c}\text { Type } 4 \text { with an } \\
\text { Ad4 head-to-tail } \\
\text { connection protein }\end{array}$ & $\begin{array}{c}\text { Number of } \\
\text { classified } \\
\text { phages }\end{array}$ & $\begin{array}{c}\text { Percentage } \\
\text { in Aclame }\end{array}$ \\
\hline Siphoviridae & 126 & 30 & & & & $156 / 169$ & 92 \\
\hline Myoviridae & 17 & $21(+7)$ & $16(+1)$ & & & $62 / 72$ & 86 \\
\hline Podoviridae & & & & $44(+3)$ & 10 & $57 / 58$ & 98 \\
\hline Unassigned & 15 & 3 & & 6 & 0 & $24 / 29$ & 83 \\
\hline Total & 158 & 61 & 17 & 53 & 10 & $299 / 328$ & 91 \\
\hline
\end{tabular}

The 299 phages in which neck proteins could be detected are detailed. The distribution of the neck proteins in each phage was interpreted in order to assign a neck architecture (or Type) to the phage. From the neck architecture and the presence or absence of a sheath protein, the morphology of the phage (Siphoviridae, Myoviridae or Podoviridae) could be deduced. For most phages, the morphology obtained from our analysis fits with that proposed by the NCBI (the 4 exceptions are discussed in the text and considered in this table as unassigned by NCBI). To highlight the consistency between our classification and that of the NCBI, we present in the following table the number of Aclame phages as a function of their proposed neck architecture (columns) and NCBI morphological class (lines). More details about the proteins detected for each phage can be found in the Additional file 2: Table S1 and on the Virfam webserver. 
are only found in Myoviridae. They comprise two headcompletion proteins homologous to T4 gp13 (noted Ad2) and T4 gp14 (noted Hc2), and a tail-completion protein homologous to T4 gp15 (noted Tc2). Finally, Type 3 (P22like), and Type 4 (Ф29-like) necks are mostly found in Podoviridae. Type 3 necks have only two head-completion proteins, homologous to P22 gp4 (noted Ad3) and gp10 (noted Hc3), and Type 4 necks have just one connection protein, homologous to $\Phi 29$ lower collar/tail tube protein gp11 (noted Ad4) [52].

\section{Using the gene context to detect additional head- and tail-completion proteins within the neck module}

At this stage, we observed that several phages could be assigned to one of the four types defined above but lacked one of the neck gene. In particular, about 13, 28 and $24 \%$ of Type 1 necks lacked an Ad1, Hc1 and Tc1, respectively, and about $85 \%$ of Type 3 necks lacked an Ad3 when using HHsearch with a confidence probability threshold of $90 \%$. We wondered whether absence of these neck proteins was real or due to, once again, the huge sequence divergence.

To further explore the existence of putative very remote homologs, we lowered the confidence probability threshold of HHsearch to $70 \%$. However, such a threshold was found too permissive in some cases and led to the detection of spurious homologs. To improve the detection specificity, we implemented an additional constraint related to the conserved head-to-tail connection module organisation observed among most of the phages (Figure 2A). In practice, the distances between the previously detected head-to-tail connection genes were calculated from their positions in the genomes. Figure $2 \mathrm{~B}$ summarizes the mean distances (and corresponding standard deviations) observed between Type 1, 2, 3 and 4 detected genes. We observed strong constraints on the relative position of the head-to-tail connection protein genes, from which we defined distance interval thresholds, corresponding to the mean +/- two standard deviations. Using these distance thresholds, we could decrease the HHsearch confidence threshold from $90 \%$ down to $70 \%$ and detect additional head-to-tail connection proteins without inclusion of spurious homologs.

As presented in Table 2, 10 Ad1, 4 Hc1, 27 Tc1 and 30 Ad3 additional proteins could be identified. In total, 747 head-to-tail connection proteins were found. We detected these proteins in 299 phages, which were unevenly distributed: Type 1 neck phages were the most prevalent (219 phages fall into this large class), while Type 2, 3 and 4 necks were found in 17, 53 and 10 phages, respectively (Figure $2 \mathrm{C}$ and Table 3 ). The specificity of our procedure was controlled by the fact that head-to-tail connection proteins were never identified in phages annotated by the NCBI as non-tailed phages: they were all detected in Caudovirales or unclassified phages. Furthermore, as already noticed, the proposed Type classes are consistent with the morphological subfamilies Siphoviridae, Myoviridae and Podoviridae. Two exceptions were identified corresponding to phages that are most probably mis-assigned in the NCBI database as deduced from recent experimental data: (i) 3 phages annotated as Siphoviridae for which we detected Podoviridae-like Ad3 and Hc3 proteins specific to Type 3 neck; these phages are Stx 1 and Stx 2 converting phages, which are closely related to $933 \mathrm{~W}$ unambiguously recognized as a Podoviridae [53,54]; (ii) 1 phage annotated in NCBI as Siphoviridae for which we detected Type 1 neck proteins and a sheath; this phage was recently reported as a Myoviridae by [55].

\section{Detection of a new $\mathrm{Ne} 1$ superfamily gene in the neck module of Type 1 phages}

We analyzed the genome organisation of the head, neck and tail proteins in the 4 neck Types, and deduced their corresponding average gene organisation (Additional file 1: Figure S1). We further explored whether any unannotated gene superfamily might emerge in the vicinity of the neck genes. Typically, in all Type 1 phages, an unannotated gene encoding a protein homologous to SPP1 gp16.1, designated hereafter $\mathrm{Ne1}$ (for neck protein of Type 1), was detected between the head and tail genes, most frequently positioned as Ad1-Hc1-Ne1-Tc1 (Ne1 is displayed in yellow in a sample of phage genomes in Figure 3). Ne1 proteins exhibit an amazing versatility of sizes, ranging from 56 to 231 residues, which probably precluded their previous identification as belonging to the same protein superfamily. However, most Ne1 proteins were detected with a HHsearch confidence threshold higher than 95\% (Additional file 1: Figure S2). The remarkable systematic presence of their gene in Type 1 neck modules suggests a critical role in the head-to-tail connection assembly or function.

Scattered experimental data exist on the function of $\mathrm{Ne} 1$ proteins. In the Siphophage $\lambda$, in the absence of the $\mathrm{Ne} 1$ protein $\mathrm{GpZ}$, viral particles are produced, but they have low infectivity [56]. GpZ was proposed to be part of the virion tail [57] and to represent a conserved family of tail proteins [58], although the systematic presence of a remote homolog in almost all Type 1 phages remained hidden due to sequence divergence. The phenotype is slightly different in mutants of the Ne1-encoding gene gpS of Myophage P2: phages form tails that are unable to attach to heads, and are non-infectious, suggesting a defect in the head-tail joining process [59]. Because of the systematic presence of the $\mathrm{Ne} 1$ gene in the neck module and of the few experimental data pointing towards an important role of $\mathrm{Ne} 1$ in virion head-to-tail connection 


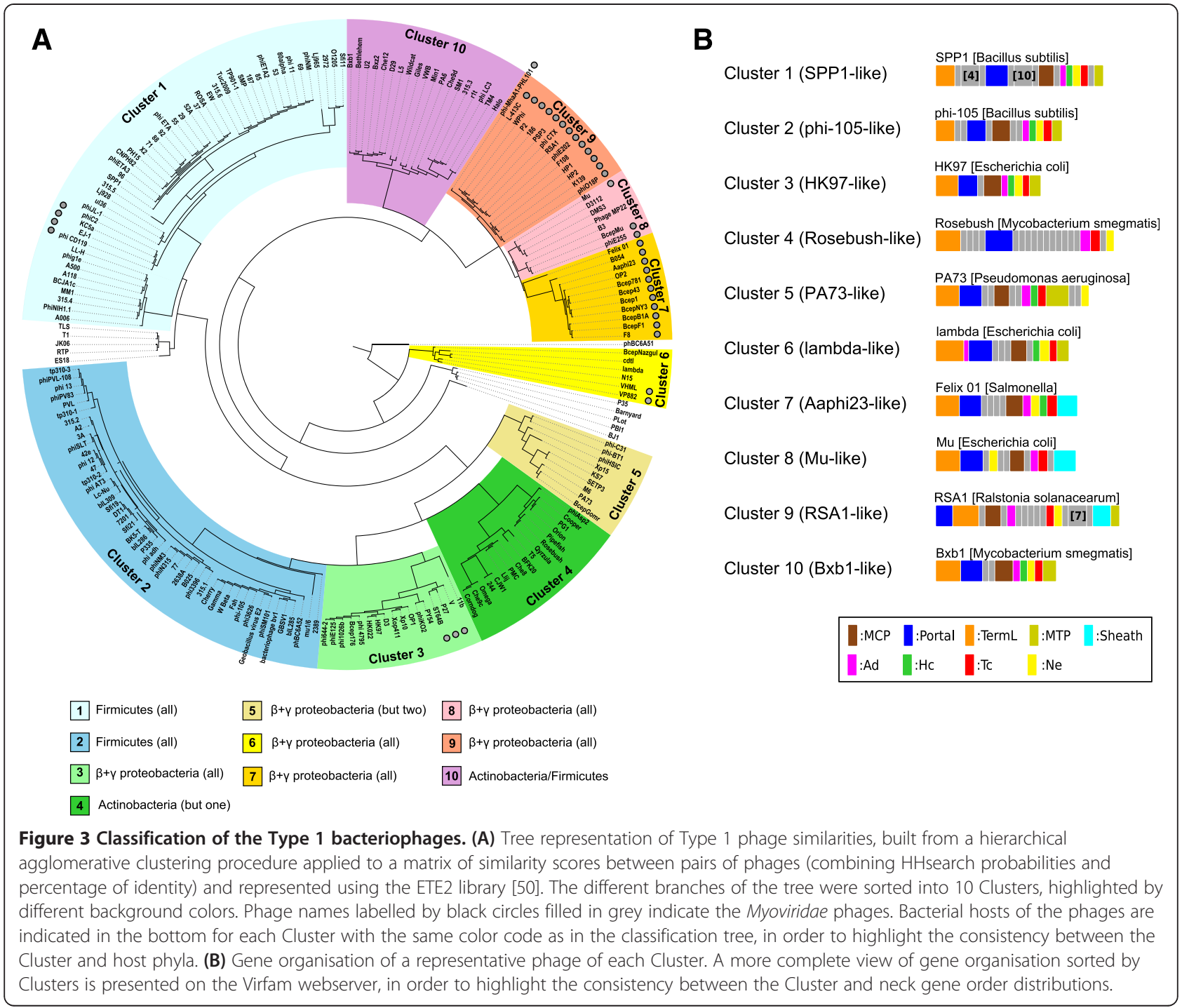

assembly and infectivity, we further included the Ne1 gene in our description of Type 1 necks (Figure 3).

\section{Phage classification from the "head-neck-tail" module analysis}

From our analysis of phage necks, we deduced that Type 1 necks are the most prevalent. Considering the large size of this group, we further subdivided it according to a metric which accounts for the "average" evolutionary divergence among the various proteins composing the defined head-neck-tail module. The probability that two protein profiles are similar as calculated by the HHsearch program is an interesting metric for quantifying the degree of remote homology between proteins. However, in case of close homology, typically above $35 \%$ of sequence identity, HHsearch probabilities peak at $100 \%$ and are not discriminative. To account for the whole spectrum of sequence divergence, we used a similarity score primarily driven by the HHsearch probability but with an additional contribution of sequence identity to properly recognize closely related phages (see Methods). For every pair of phages, similarity scores were calculated between all components of the head-neck-tail module (comprising up to 9 proteins) and then averaged to yield a mean score reflecting the similarity between the head-neck-tail modules of the phage pair. From this metric, a hierarchical agglomerative clustering procedure was used to obtain a tree representation of phage similarities (Figure 3A; this analysis is extended to Types 2-4 phages on the Virfam webserver).

The clustering procedure discriminated ten major Clusters within the Type 1 phage family. Inside each Cluster, HHsearch probabilities between homologous proteins are often close to $100 \%$, but even in these cases, identities between protein sequences can be very low (in the range 10-20\%) (analysis of a representative subset of phages of Type 1 Cluster 1 in Additional file 1: 
Figure S3A; similar analyses are available on other Types and Clusters on the Virfam webserver). Between distinct Clusters, HHsearch mean probabilities are on average below $50 \%$, and the connection between these groups is achieved through a limited number of proteins bridging together the whole ensemble (Additional file 1: Figure S3B and the Virfam webserver, by clicking on the Type or Cluster of interest at http://biodev.cea.fr/virfam/ tables_results/help/AllTypes.html).

As illustrated in Figure 3A, the Clusters group together phages that infect hosts from the same phylogenetic clades supporting the consistency of the classification. A characteristic of Type 1 phage is that their hosts spread over the whole bacterial phylogenetic tree which is not the case for all the Types defined in our study (see Discussion). Also, genome organization of the head-neck-tail module is partitioned in a homogeneous manner among the Clusters although no prior constraint related to gene order or to inter-gene distance was included in the clustering metrics (Figure 3B; see also the Virfam webserver). An additional remarkable feature standing out from this classification is that phages from the Myoviridae family do not tend to cluster together. Phages with a sheath in their genome are spread over more than half of the Clusters, namely Clusters $1,3,6,7,8$ and 9 , together with closely related phages deprived from a contractile system. Such versatility in the presence/absence of a sheath protein had previously been noticed in the case of phages related to $\mathrm{Mu}$ (grouped in Cluster 8) [15] and appears as a general property of Type 1 phages.

\section{Automatic identification of neck proteins and classification of necks in tailed bacteriophages through the Virfam server}

Our analysis and resulting classification enabled to classify 299 phages within 328 tailed and unclassified Aclame phages. To enlarge our study to newly sequenced phages, we built the Virfam webserver interface (Figure 4). We challenged the robustness and representativeness of our Webserver by investigating 624 new phage genomes available at the NCBI (July 2013 update). Within these phages, 19 are annotated as non-tailed phages, and indeed could not be classified by our approach. The 605 remaining phages are either described as Caudovirales $(n=601)$ or unclassified by the NCBI $(n=4)$. 93\% of these phages could be sorted among the four neck Types by our approach (Table 4). Interestingly, the distribution of the different Types of phages is similar to that observed in the learning phage database. Some phage subfamilies are enriched. For example, at least 7 N4-like phages [60] are now found within Type 3 neck phages, and they all lack Hc3, thus confirming that the head-closure protein of N4-like phages is highly divergent. In the case of Type 1 phages, the distribution within Clusters is also similar to that observed for the learning set: $89 \%$ of these phages could be assigned to one of the previously defined Clusters. We conclude that the classification constructed on the 299 Aclame phage genomes is relevant for a global classification of most of the 624 newly analyzed genomes. The Virfam server is thus a robust tool for the classification of novel tailed phages and the identification of their structural organization at the head-to-tail interface.

\section{Discussion}

Understanding the genomic and structural diversity of phages is a critical challenge, stimulated by the increasing number of available phage sequences. $96 \%$ of the bacteriophages isolated so far are tailed bacteriophages. Here, we described the structural diversity of a set of proteins essential for tailed phage virion assembly and function, comprising both the semi-conserved capsid, portal, terminase, major tail and sheaths proteins and the more divergent head-to-tail connection proteins. Neck proteins are particularly important for capsid completion, hence for the phage life cycle: they form a channel between the head and the tail, that has to close rapidly after DNA packaging in order to avoid DNA leakage and to reopen after recognition of the targeted bacteria in order to allow infection. Despite their diversity, they are thus submitted to significant evolutionary constraints which we attempted to reveal by combining profile-profile comparisons with gene context analyses. Our ambition was to evaluate the protein sequence and composition diversity at the head-to-tail connection and to use this knowledge to propose a detailed phage classification related to phage morphology.

\section{Robustness of the proposed classification}

We first successfully detected a large set of structural proteins present in 299 phages, comprising 92\% of the Siphoviridae, $86 \%$ of the Myoviridae and $98 \%$ of the Podoviridae of our learning database (Additional file 2: Table S1). We identified a Type and thus a neck architecture for these phages, and proposed a classification within phages of the same Type and thus with the same neck structure. This approach enabled classification of 91\% of the Caudovirales and unclassified phages of the learning database and 93\% of the Caudovirales and unclassified phages of the NCBI database (Additional file 3: Table S2). The higher success of our procedure in the case of the NCBI database is linked to an overrepresentation of Type 2 phages, which are more closely related and thus easier to detect.

Interestingly, we find that the resulting phage classification is correlated to the targeted bacteria phylogenetic tree: if Type 1 phages infect a very large number of hosts spanning the whole bacterial phylogenetic tree and in 


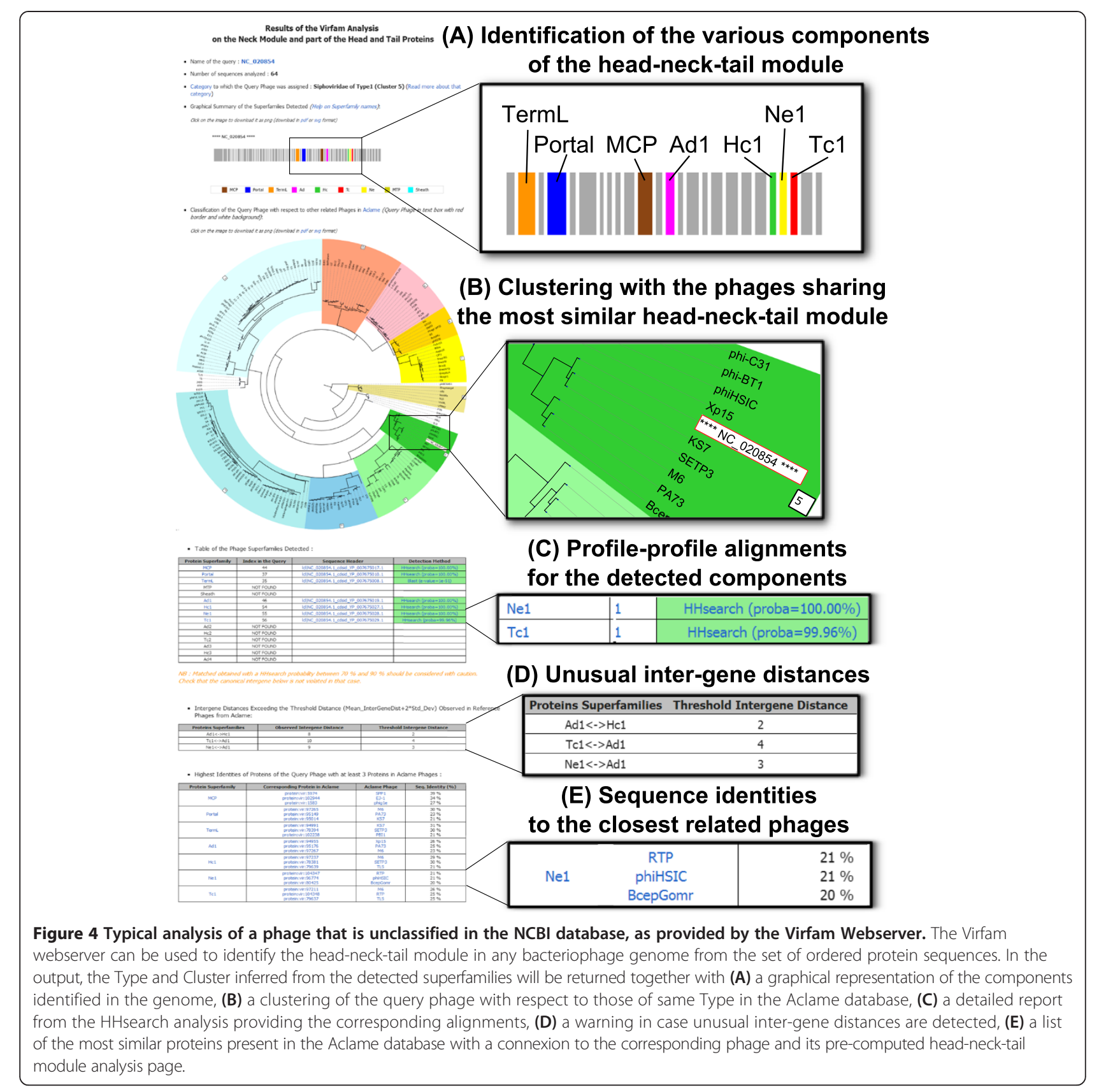

Table 4 Neck modules detected through the Web server in phages classified in the NCBI database but not in Aclame

\begin{tabular}{lcccccc}
\hline & Type 1 & Type 2 & Type 3 & Type 4 & Total & Percentage in NCBI \\
\hline Siphoviridae & 278 & & & & $278(244$ annotated as Siphoviridae $)$ & 94 \\
Myoviridae & 81 & 75 & & & $156(138$ annotated as Myoviridae $)$ & 85 \\
Podoviridae & & 116 & 10 & $126(101$ annotated as Podoviridae $)$ & 99 \\
Total & 359 & 75 & 116 & 10 & 560 & 93 \\
\hline
\end{tabular}

Percentages are calculated within the Siphoviridae, Myoviridae and Podoviridae subfamilies described by the NCBI. Total percentage corresponds to the number of assigned phages (560) compared to the total number of Caudovirales and unclassified phages of the analyzed NCBI database (624-19 non tailed bacteriophages $=605)$. 
particular Cyanobacteria, Proteobacteria and Firmicutes, Type 2 and Type 3 are only found so far to infect Cyanobacteria and Proteobacteria and Type 4 are only detected as infecting Firmicutes (Additional file 1: Figure S4).

It was proposed that within the neck, the Portal protein alone is already an efficient marker for phage classification as a function of virion architecture (Comeau et al. [61]). Using our scoring function calculated on the Portal protein alone, we built a tree of all the Aclame phages (Additional file 1: Figure S5A). The resulting Type classification is similar to that described in our study (only two Type 3 phages were not correctly clustered with the remaining Type 3 phages). Using the three MCP, Portal and TermL protein markers did not improve the discrimination of Types (Additional file 1: Figure S5B). We also observed an overall good consistency between the Portal classification and our Type 1 tree generated using multiple markers.

However, significant discrepancy is observed in the phages hosted by Actinobacteria (mainly Mycobacteria), which were found to group in Clusters 4 and 10 in our Type 1 multi-protein classification. If only the Portal is used, many phages populating Cluster 4 are spread in different branches of the tree irrespective of the nature of their hosts (highlighted by a mark in Additional file 1: Figure S5A). Moreover, phages Giles and Min1 that were connected to Cluster 10 are not anymore clustered with phages from Actinobacteria. Therefore, observations on highly divergent phages from Podoviridae or from Clusters 4 and 10 show that the strategy we propose, using neck proteins, is able to discriminate phages hosted by bacteria from the same Phylum. The issue noticed for members of Clusters 4 and 10 can also be perceived for Cluster 3 (hosted by Proteobacteria) which tends to mix within cluster 2 (hosted by Firmicutes) in the Portalonly tree (Additional file 1: Figure S5A). Altogether our results show that the Portal protein may diverge in evolution while head-to-tail connection proteins still show consistent evolutionary links with proteins from other phages (Figure 5). The metric used to establish the phage similarity matrix account for that property. The fact that our multi-protein classification in four Types is (i) globally consistent with Portal-only classification (ii) successful in difficult cases in recognizing phages hosted in Bacteria of the same phylum supports the idea that neck proteins bring useful signal to the classification of phages.

\section{Comparison to previously described whole genome classifications}

Previous studies have shown that Podoviridae and Myoviridae could be classified using BLAST-based tools and phage distance calculations based on global shared genes $[9,14,61]$. These phages were divided into subfamilies, which were recognized as biologically significant and are available on the ICTV website [62]. Podoviridae were classified into two main subfamilies, Picovirinae (comprising Ф29-like phages and several other genera) that corresponds to the present Type 4, and Autographivirinae (comprising T7-like phages and several other genera) that perfectly matches with one branch of our Type 3 (Additional file 1: Figure S6). Other groups of the Type 3 classification are close to the tentative P22containing subfamily and to several of the unclassified genera (Additional file 1: Figure S3). Hence, our analysis connects in a hierarchical and consistent manner several independent groups of Podoviridae, such as Autographivirinae and P22-like phages, on the basis of the similarities between their head-to-tail modules.

The ICTV classification of Myoviridae is more difficult to overlap with ours. These phages were previously classified into three main subfamilies, Peduovirinae (comprising P2-like and HP1-like phages), Teequatrovirinae (comprising T4-like phages and several other genera) and Spounavirinae (comprising SPO1-like and Twort-like phages) $[9,61]$, plus several other independent genera. The Teequatrovirinae subfamily corresponds to the Type 2 category of our classification. But in our Type 1 class, Myoviridae from the other subfamilies or genera are often mixed with Siphoviridae in Type 1 Clusters, suggesting that their contractile character has been acquired or lost in a versatile manner throughout phage evolution. Consistent clusters were however retrieved within these myophages. P2-like, $\mathrm{Mu}$-like and AaФ23-like phages that were described as very different, based on BLAST- analyses $[9,61]$ are well partitioned into different Type 1 clusters. Peduovirinae perfectly matches Type 1 Cluster 9, while four previously independent genera of Myoviridae are now grouped into Type 1 Cluster 7. Finally, the Spounavirinae subfamily is left as a special category in which only one Ad1 component could be identified but no Hc or Tc subunits recognized so far. Although recognized as Type 1-like, they could not be further clustered and will be interesting to investigate further.

Altogether, our automatic classification tool consistently retrieved the known phage subfamilies as they were described for Podoviridae and Myoviridae. The remote homology analysis further provides evidence on how to connect independent subgroups based on their neck architecture similarities. Moreover, it supports a classification for the Siphoviridae and highlights the neck structural relationships between Sipho- and Myoviridae.

\section{A common structural core for all neck Types?}

Our classification highlights the representativeness of four different Types of neck architecture. Each Type is characterized by a set of specific head-to-tail connection proteins. It is also characterized by a specific distribution of phage genome sizes. Tiny phages (encoded by less than 30 


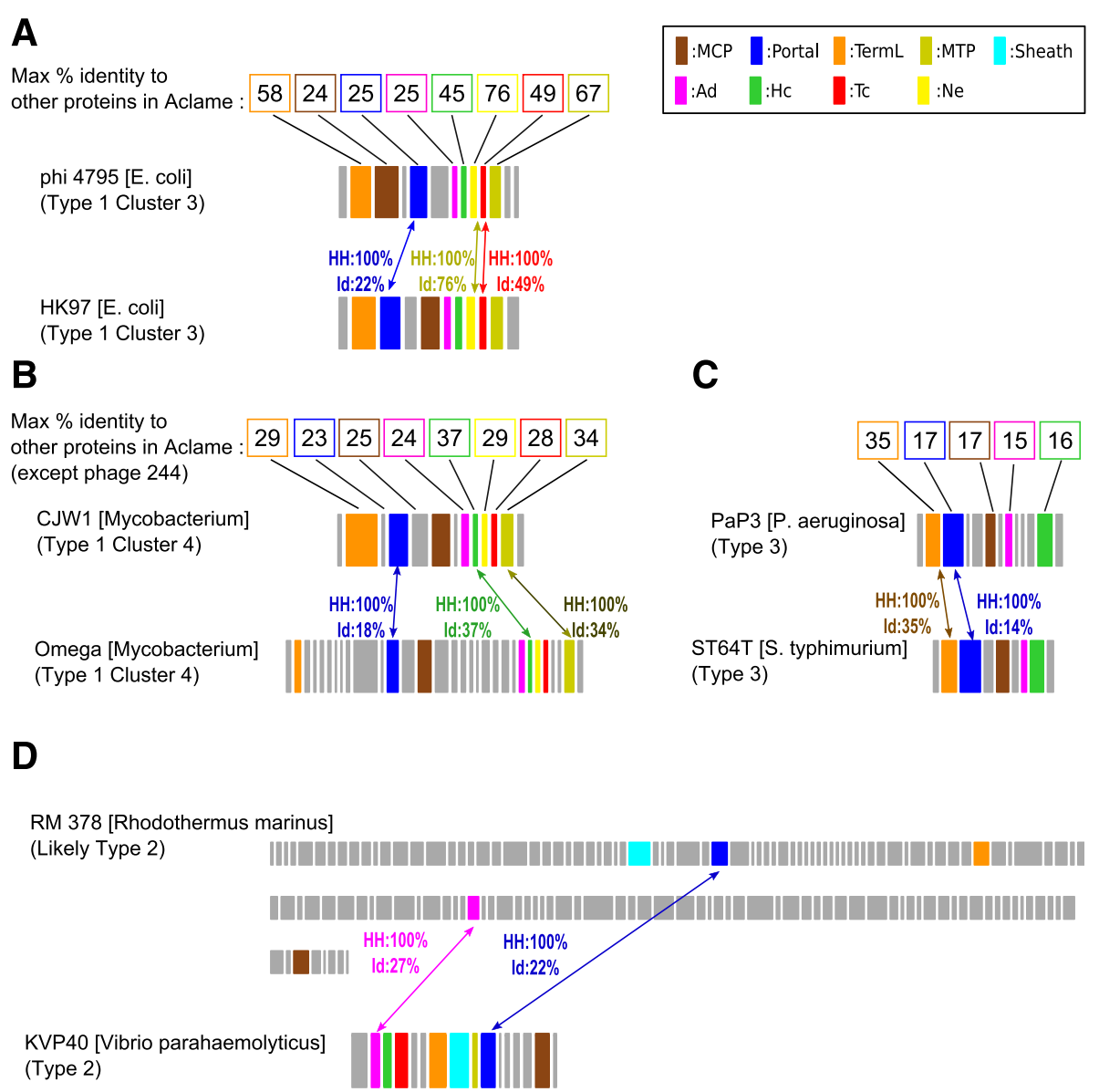

Figure 5 Interest of using a multi-protein analysis for phage classification when Portal sequence diverged more than other proteins of the head-neck-tail module. Four pairwise comparisons between the head-neck-tail modules of phages whose Portal proteins have significantly diverged with respect to other proteins of the head-neck-tail module. (A) Phage phi 4795, assigned to Type 1 Cluster 3, has a Portal sharing less than 25\% identity with any other protein in Aclame database. Its TermL, Ne1, Tc1 and MTP proteins present a significantly higher conservation profile. In particular, compared with HK97, Ne1 and Tc1 share 76\% and 49\% identity with their homologs in phi 4795, respectively, while Portal only shares 22\%. (B) Phage CJW1, assigned to Type 1 Cluster 4, has a Portal sharing less than 23\% identity with any other protein in Aclame database. Proteins such as Hc1 or MTP share a higher conservation profile. In particular, they share 37\% and 34\% identity with $\mathrm{Ne} 1$ and Tc1 of phage Omega, respectively while the Portal only shares $18 \%$. (C) Phage PaP3, assigned to Type 3, has a Portal sharing less than 17\% identity with any of the other proteins of Aclame database. In contrast, its TermL diverged to a lesser extent sharing 35\% identity with that of phage ST64T. (D) RM 378 is among the most divergent phages assigned to Type 2. Only an Ad2 protein could be detected when searching for its head-to-tail connection proteins. Although its Portal shares at most $22 \%$ identity with the closest Portal of other phages of Aclame, its Ad2 protein could be recognized with higher identity (27\% identity with KVP40) providing stronger support for the assignment of this phage to the Type 2 category.

genes) generally exhibit Type 4 necks, common size phages (encoded by 31 to 150 genes) present either Type 1 or Type 3 necks, and large phages often show Type 2 necks (Additional file 1: Figure S7 and Additional file 1: Text S1). Physical explanations might be discovered in the future that explain this correlation between neck proteins and genome size. Interestingly, our scoring of phage protein homologies also suggests that the different neck Types might share some common structural properties. Indeed, structural analogies exist within each category of neck components, the so-called Ad, Hc and Tc. For instance all four Ad1, Ad2, Ad3 and Ad4 exhibit 4 to 5 predicted $\alpha$-helices and HHsearch confidently predicts homology between an Ad1 and an Ad3 (Additional file 1: Text S2). Comparison between Ad1 and Ad3 protein structures consistently revealed that they share the same $\alpha$-helical bundle fold (gp15 of SPP1 [63], gp6 of HK97 [64] for Ad1 and gp4 of P22 for Ad3 [65]). Similar observations were made on the $\mathrm{Hc} 1, \mathrm{Hc} 2$ and $\mathrm{Hc} 3$ proteins: they are all predicted to fold into a $\beta$ - strand rich structure and HHsearch suggests homology between a Hc1 and a Hc2 (Additional file 1: Text S2). As regards Tc1 and Tc2, secondary structure prediction and our HHsearch calculations again predict that they share a common structural core. Moreover, recent determination of the three-dimensional structure of a Tc2 protein 
(gp15 from phage T4) uncovers its structural analogy with a Tc1 protein, gpU from phage $\lambda$ Fokine et al. [66]. Altogether, these observations support the existence of structural similarities between the components of the various neck types classified in this work and advocate for the existence of a common, highly remote, virion ancestor showing neck structural characteristics close to those highlighted in this study. Given the huge diversity of Type 1 phages highlighted in this work, their ability to populate all bacterial phyla and some archaeal ones (data not shown), these phages are well suited to represent the ancestral neck organization in Caudovirales.

\section{Conclusions}

Through the Virfam webserver, it is now possible to detect both the relatively well-conserved capsid, large terminase and tail genes and the highly diverse head-to-tail connection genes. Differences at the head-to-tail connection in protein number and fold were highlighted within tailed phages, and large differences were observed between phage subfamilies (Siphoviridae, Myoviridae, Podoviridae). We showed that these proteins are crucial to define the type of morphology a phage likely adopts. We reasoned that going further into the description of the phage head-to-tail differences should provide a classification based on detailed virion morphology. Our study demonstrated that a combination of sequence and profile-profile comparison scores can be used to delineate consistent subgroups in the remote homology space of Caudovirales virions. Given the high detection rate of the neck genes provided by Virfam, most new phages can now be positioned within a "head-neck-tail module based" classification. Outside this specific module it is clear that each phage has also evolved and adapted to its specific environment. Versatility and reshuffling of gene functions in phage genomes, which often confuse a phylogenetic classification of phages, may have been less intense in the head-neck-tail module. This module may thus serve as a probe for further exploring the evolution of phages.

Our methodology enables classification and comparison of viromes. Terminases and major tail proteins were previously proposed as phage markers. Unlike these single gene approaches, our characterisation of the phage headneck-tail module is not hindered when one of the genes is particularly divergent, and this gene can even be detected, provided its genome position is canonical. Identification of neck genes within a phage genome further suggests that this genome codes for a functional virion, thus differentiating phages and other mobile genetic elements. Altogether, as the Virfam server allows for an automatic classification of phages, it should facilitate assignment of viromes and detection of functional phages from bacterial metagenomic samples.

\section{Availability and requirements}

The Virfam webserver is accessible at the following address: http://biodev.extra.cea.fr/virfam/. The input is a phage genome. The order of the genes must be preserved because it is used in our analysis. Further information on the input format is available by clicking on the "how to proceed" button of the main page.

\section{Additional files}

\begin{abstract}
Additional file 1: Figure S1. Mean arrangement of the neck genes relatively to the head and tail genes in Type 1 Siphoviridae, Type 1 Myoviridae, Type 2 Myoviridae, Type 3 Podoviridae and Type 4 Podoviridae: (A) Inter-genedistance matrices resulting from the analysis of the Aclame genomes, (B) Graphical representation ofthe average gene organization for each neck Type. Figure S2. Secondary structure analysis of Type 1 neck Ne1 proteins identified in (A) Siphoviridae and (B) Myoviridae. Figure S3. Matrices of profile-profile comparison and identity scores (A) within a typical Type 1 Cluster 1 and (B) between Type 1 Clusters. Figure S4. Relationship between the identified phage neck Types and the host bacteria phyla. Figure S5. Global tree calculated using only (A) the Portal or (B) the MCP-TermL-Portal triad to compute the similarity matrix between phages. Figure S6. Tree representation of the clustering applied to bacteriophages belonging to the Type2, Type3 and Type4. Figure S7. Distribution of the Aclame phages of known morphology as a function of their genome size and neck Type. Text S1. Relationship between neck structural organisation and genome size in tailed bacteriophages. Text S2. Structural analogies between the different neck Types. Method S1. Procedure to assess the sensitivity of PSI-Blast search for Table 2. Tables S3. Profile identifiers used to test the procedure of remote homology detection using PSI-Blast.
\end{abstract}

Additional file 2: Table S1. Table of the 447 phages from Aclame with all proteins assigned by the virfam strategy.

Additional file 3: Table S2. Table of the 623 most recent phages from the NCBI with all proteins assigned by the virfam strategy.

\section{Competing interests}

The authors declare that they have no competing interests.

\section{Authors' contributions}

AL encoded the bioinformatics approach and analyzed the results, PT and MAP contributed to the data analysis, RG designed and encoded the bioinformatics approach and contributed to the bioinformatics analysis. SZJ conceived the project, analyzed data, supervised the entire project. MAP, RG and SZJ wrote the manuscript. All authors read and approved the final manuscript.

\section{Acknowledgements}

We thank Ariane Toussaint for very fruitful discussions, Jessica Andréani for carefully rereading the manuscript and both anonymous reviewers for their insightful comments. This work was supported by the French Infrastructure for Integrated Structural Biology [ANR-10-INSB-05-01] and by the French National Research Agency [ANR grant "DNA Gating" to S.Z.J. and P.T., ANR grant "Dynamophage" to R.G. and M.A.P.]

\section{Author details}

${ }^{1}$ CEA, iBiTecS, Gif-sur-Yvette, F-91191 Paris, France. ${ }^{2}$ Université Paris-Sud \& CNRS, UMR 8221, Gif-sur-Yvette, F-91191 Paris, France. ${ }^{3}$ Present address: Institut de Génétique et Microbiologie, Université Paris-Sud, Orsay, Paris, France. ${ }^{4}$ Unité de Virologie Moléculaire et Structurale, UMR CNRS 2472, UMR INRA 1157 and IFR 115, Gif-sur-Yvette, Paris, France. ${ }^{5}$ INRA, UMR1319, Micalis, domaine de Vilvert, Jouy en Josas, Paris, France. ${ }^{\circ}$ AgroParisTech, UMR1319, Micalis, domaine de Vilvert, Jouy en Josas, Paris, France.

Received: 17 June 2014 Accepted: 29 October 2014

Published: 27 November 2014 


\section{References}

1. Krupovic M, Prangishvili D, Hendrix RW, Bamford DH: Genomics of bacterial and archaeal viruses: dynamics within the prokaryotic virosphere. Microbiol Mol Biol Rev 2011, 75(4):610-635.

2. Ackermann HW: Phage or phages. Bacteriophage 2011, 1(1):52-53.

3. Rohwer F: Global phage diversity. Cell 2003, 113(2):141.

4. Angly FE, Felts B, Breitbart M, Salamon P, Edwards RA, Carlson C, Chan AM, Haynes M, Kelley S, Liu H, Mahaffy JM, Mueller JE, Nulton J, Olson R, Parsons R, Rayhawk S, Suttle CA, Rohwer F: The marine viromes of four oceanic regions. PLoS Biol 2006, 4(11):e368.

5. Sunagawa S, Mende DR, Zeller G, Izquierdo-Carrasco F, Berger SA, Kultima JR, Coelho LP, Arumugam M, Tap J, Nielsen HB, Rasmussen S, Brunak S, Pedersen O, Guarner F, de Vos WM, Wang J, Li J, Doré J, Ehrlich SD, Stamatakis A, Bork P: Metagenomic species profiling using universal phylogenetic marker genes. Nat Methods 2013, 10(12):1196-1199.

6. Ackermann HW: 5500 Phages examined in the electron microscope. Arch Virol 2007, 152(2):227-243.

7. Lawrence JG, Hatfull GF, Hendrix RW: Imbroglios of viral taxonomy: genetic exchange and failings of phenetic approaches. J Bacteriol 2002, 184(17):4891-4905.

8. Rohwer F, Edwards R: The Phage Proteomic Tree: a genome-based taxonomy for phage. J Bacteriol 2002, 184(16):4529-4535.

9. Lavigne R, Darius P, Summer EJ, Seto D, Mahadevan P, Nilsson AS, Ackermann HW, Kropinski AM: Classification of Myoviridae bacteriophages using protein sequence similarity. BMC Microbio/ 2009, 9:224

10. Proux C, van Sinderen D, Suarez J, Garcia P, Ladero V, Fitzgerald GF, Desiere $\mathrm{F}$, Brussow H: The dilemma of phage taxonomy illustrated by comparative genomics of Sfi21-like Siphoviridae in lactic acid bacteria. J Bacteriol 2002, 184(21):6026-6036.

11. Serwer P, Hayes SJ, Zaman S, Lieman K, Rolando M, Hardies SC: Improved isolation of undersampled bacteriophages: finding of distant terminase genes. Virology 2004, 329(2):412-424.

12. Sullivan MB, Coleman ML, Quinlivan V, Rosenkrantz JE, Defrancesco AS, Tan G, Fu R, Lee JA, Waterbury JB, Bielawski JP, Chisholm SW: Portal protein diversity and phage ecology. Environ Microbiol 2008, 10(10):2810-2823.

13. Smith KC, Castro-Nallar E, Fisher JN, Breakwell DP, Grose JH, Burnett SH: Phage cluster relationships identified through single gene analysis. BMC Genomics 2013, 14(1):410.

14. Lavigne R, Seto D, Mahadevan P, Ackermann HW, Kropinski AM: Unifying classical and molecular taxonomic classification: analysis of the Podoviridae using BLASTP-based tools. Res Microbiol 2008, 159(5):406-414.

15. Lima-Mendez G, Van Helden J, Toussaint A, Leplae R: Reticulate representation of evolutionary and functional relationships between phage genomes. Mol Biol Evol 2008, 25(4):762-777.

16. Veesler D, Cambillau C: A common evolutionary origin for tailedbacteriophage functional modules and bacterial machineries. Microbiol Mol Biol Rev 2011, 75(3):423-433. first page of table of contents.

17. Cuervo A, Carrascosa JL: Viral connectors for DNA encapsulation. Curr Opin Biotechnol 2011, 23(1):1-8.

18. Tavares P, Zinn-Justin S, Orlova EV: Genome Gating in Tailed Bacteriophage Capsids. New-York: Springer; 2011.

19. Pell LG, Kanelis V, Donaldson LW, Howell PL, Davidson AR: The phage lambda major tail protein structure reveals a common evolution for long-tailed phages and the type VI bacterial secretion system. Proc Natl Acad Sci U S A 2009, 106(11):4160-4165.

20. Sun S, Rao VB, Rossmann MG: Genome packaging in viruses. Curr Opin Struct Biol 2010, 20(1):114-120.

21. Hendrix RW: Symmetry mismatch and DNA packaging in large bacteriophages. Proc Natl Acad Sci U S A 1978, 75(10):4779-4783.

22. Lurz R, Orlova EV, Gunther D, Dube P, Droge A, Weise F, van Heel M, Tavares P: Structural organisation of the head-to-tail interface of a bacterial virus. J Mol Biol 2001, 310(5):1027-1037.

23. Orlova EV, Gowen B, Droge A, Stiege A, Weise F, Lurz R, van Heel M, Tavares P: Structure of a viral DNA gatekeeper at $10 \mathrm{~A}$ resolution by cryo-electron microscopy. EMBO J 2003, 22(6):1255-1262.

24. Xiang Y, Morais MC, Battisti AJ, Grimes S, Jardine PJ, Anderson DL, Rossmann MG: Structural changes of bacteriophage phi29 upon DNA packaging and release. EMBO J 2006, 25(21):5229-5239.

25. Tang J, Lander GC, Olia AS, Li R, Casjens S, Prevelige P Jr, Cingolani G, Baker TS, Johnson JE: Peering down the barrel of a bacteriophage portal: the genome packaging and release valve in p22. Structure 2011, 19(4):496-502.
26. Edmonds L, Liu A, Kwan JJ, Avanessy A, Caracoglia M, Yang I, Maxwell KL, Rubenstein J, Davidson AR, Donaldson LW: The NMR structure of the gpU tail-terminator protein from bacteriophage lambda: identification of sites contributing to $\mathrm{Mg}$ (II)-mediated oligomerization and biological function. J Mol Biol 2007, 365(1):175-186.

27. Chagot B, Auzat I, Gallopin M, Petitpas I, Gilquin B, Tavares P Zinn-Justin S: Solution structure of gp17 from the Siphoviridae bacteriophage SPP1: insights into its role in virion assembly. Proteins 2012, 80(1):319-326.

28. Leiman PG, Chipman PR, Kostyuchenko VA, Mesyanzhinov W, Rossmann MG: Three-dimensional rearrangement of proteins in the tail of bacteriophage T4 on infection of its host. Cell 2004, 118(4):419-429.

29. Lopes A, Amarir-Bouhram J, Faure G, Petit MA, Guerois R: Detection of novel recombinases in bacteriophage genomes unveils Rad52, Rad51 and Gp2.5 remote homologs. Nucleic Acids Res 2010, 38(12):3952-3962.

30. Leplae R, Lima-Mendez G, Toussaint A: ACLAME: A CLAssification of Mobile genetic Elements, update 2010. Nucleic Acids Res 2010, 38(Database issue):D57-D61.

31. Altschul SF, Madden TL, Schaffer AA, Zhang J, Zhang Z, Miller W, Lipman DJ: Gapped BLAST and PSI-BLAST: a new generation of protein database search programs. Nucleic Acids Res 1997, 25(17):3389-3402.

32. Söding J: Protein homology detection by HMM-HMM comparison. Bioinformatics 2005, 21(7):951-960.

33. Jones DT: Protein secondary structure prediction based on position-specific scoring matrices. J Mol Biol 1999, 292(2):195-202.

34. Fokine A, Leiman PG, Shneider MM, Ahvazi B, Boeshans KM, Steven AC, Black LW, Mesyanzhinov W, Rossmann MG: Structural and functional similarities between the capsid proteins of bacteriophages T4 and HK97 point to a common ancestry. Proc Natl Acad Sci U S A 2005, 102(20):7163-7168.

35. Wikoff WR, Liljas L, Duda RL, Tsuruta H, Hendrix RW, Johnson JE: Topologically linked protein rings in the bacteriophage HK97 capsid. Science 2000, 289(5487):2129-2133.

36. Conway JF, Wikoff WR, Cheng N, Duda RL, Hendrix RW, Johnson JE, Steven AC: Virus maturation involving large subunit rotations and local refolding. Science 2001, 292(5517):744-748.

37. Gertsman I, Gan L, Guttman M, Lee K, Speir JA, Duda RL, Hendrix RW, Komives EA, Johnson JE: An unexpected twist in viral capsid maturation. Nature 2009, 458(7238):646-650.

38. White HE, Sherman MB, Brasiles $S$, Jacquet E, Seavers $P$, Tavares $P$, Orlova EV: Capsid structure and its stability at the late stages of bacteriophage SPP1 assembly. J Virol 2012, 86(12):6768-6777.

39. Olia AS, Bhardwaj A, Joss L, Casjens S, Cingolani G: Role of gene 10 protein in the hierarchical assembly of the bacteriophage $\mathrm{P} 22$ portal vertex structure. Biochemistry 2007, 46(30):8776-8784.

40. Lebedev AA, Krause MH, Isidro AL, Vagin AA, Orlova EV, Turner J, Dodson EJ, Tavares $P$, Antson AA: Structural framework for DNA translocation via the viral portal protein. EMBO J 2007, 26(7):1984-1994.

41. Alam TI, Draper B, Kondabagil K, Rentas FJ, Ghosh-Kumar M, Sun S, Rossmann MG, Rao VB: The headful packaging nuclease of bacteriophage T4. Mol Microbiol 2008, 69(5):1180-1190.

42. Sun S, Kondabagil K, Draper B, Alam TI, Bowman VD, Zhang Z, Hegde S, Fokine A, Rossmann MG, Rao VB: The structure of the phage T4 DNA packaging motor suggests a mechanism dependent on electrostatic forces. Cell 2008, 135(7):1251-1262.

43. Smits C, Chechik M, Kovalevskiy OV, Shevtsov MB, Foster AW, Alonso JC, Antson AA: Structural basis for the nuclease activity of a bacteriophage large terminase. EMBO Rep 2009, 10(6):592-598.

44. Burroughs $A M$, lyer $L M$, Aravind $L$ : Comparative genomics and evolutionary trajectories of viral ATP dependent DNA-packaging systems. Genome Dyn 2007, 3:48-65.

45. Söding J, Biegert A, Lupas AN: The HHpred interactive server for protein homology detection and structure prediction. Nucleic Acids Res 2005, 33(Web Server issue):W244-W248

46. Aksyuk AA, Leiman PG, Kurochkina LP, Shneider MM, Kostyuchenko VA, Mesyanzhinov W, Rossmann MG: The tail sheath structure of bacteriophage T4: a molecular machine for infecting bacteria. EMBO J 2009, 28(7):821-829.

47. Aksyuk AA, Kurochkina LP, Fokine A, Forouhar F, Mesyanzhinov W, Tong L, Rossmann MG: Structural conservation of the myoviridae phage tail sheath protein fold. Structure 2011, 19(12):1885-1894. 
48. Katsura I, Tsugita A: Purification and characterization of the major protein and the terminator protein of the bacteriophage lambda tail. Virology 1977, 76(1):129-145.

49. Casjens S, Horn T, Kaiser AD: Head assembly steps controlled by genes $\mathrm{F}$ and W in bacteriophage lambda. J Mol Biol 1972, 64(3):551-563.

50. Huerta-Cepas J, Dopazo J, Gabaldon T: ETE: a python Environment for Tree Exploration. BMC Bioinformatics 2010, 11:24.

51. Maxwell KL, Yee AA, Booth V, Arrowsmith CH, Gold M, Davidson AR: The solution structure of bacteriophage lambda protein W, a small morphogenetic protein possessing a novel fold. J Mol Biol 2001, 308(1):9-14.

52. Tang J, Olson N, Jardine PJ, Grimes S, Anderson DL, Baker TS: DNA poised for release in bacteriophage phi29. Structure 2008, 16(6):935-943.

53. Garcia-Aljaro C, Muniesa M, Jofre J, Blanch AR: Genotypic and phenotypic diversity among induced, stx2-carrying bacteriophages from environmental Escherichia coli strains. Appl Environ Microbiol 2009, 75(2):329-336.

54. Rooks DJ, Libberton B, Woodward MJ, Allison HE, McCarthy AJ: Development and application of a method for the purification of free shigatoxigenic bacteriophage from environmental samples. J Microbiol Methods 2012, 91(2):240-245.

55. Dorscht J, Klumpp J, Bielmann R, Schmelcher M, Born Y, Zimmer M, Calendar R, Loessner MJ: Comparative genome analysis of Listeria bacteriophages reveals extensive mosaicism, programmed translational frameshifting, and a novel prophage insertion site. J Bacteriol 2009, 191(23):7206-7215.

56. Casjens $\mathrm{S}$, Hendrix R: Comments on the arrangement of the morphogenetic genes of bacteriophage lambda. J Mol Biol 1974, 90(1):20-25.

57. Katsura I: Mechanism of length determination in bacteriophage lambda tails. Adv Biophys 1990, 26:1-18.

58. Davidson AR, Cardarelli L, Pell LG, Radford DR, Maxwell KL: Long noncontractile tail machines of bacteriophages. Adv Exp Med Biol 2012, 726:115-142.

59. Lengyel JA, Goldstein RN, Marsh M, Calendar R: Structure of the bacteriophage P2 tail. Virology 1974, 62(1):161-174.

60. Fouts DE, Klumpp J, Bishop-Lilly KA, Rajavel M, Willner KM, Butani A, Henry M, Biswas B, Li M, Albert MJ, Loessner MJ, Calendar R, Sozhamannan S: Whole genome sequencing and comparative genomic analyses of two Vibrio cholerae 0139 Bengal-specific Podoviruses to other N4-like phages reveal extensive genetic diversity. Virol J 2013, 10:165.

61. Comeau AM, Tremblay D, Moineau S, Rattei T, Kushkina Al, Tovkach FI, Krisch HM, Ackermann HW: Phage morphology recapitulates phylogeny: the comparative genomics of a new group of myoviruses. PLoS One 2012, 7(7):e40102.

62. King AMQ, Adams MJ, Carstens EB, Lefkowitz EJ (Eds): Virus Taxonomy: Classification and Nomenclature of Viruses: Ninth Report of the International Committee on taxonomy of Viruses. San Diego: Elsevier Academic Press; 2012.

63. Lhuillier S, Gallopin M, Gilquin B, Brasiles S, Lancelot N, Letellier G, Gilles M, Dethan G, Orlova EV, Couprie J, Tavares P, Zinn-Justin S: Structure of bacteriophage SPP1 head-to-tail connection reveals mechanism for viral DNA gating. Proc Natl Acad Sci U S A 2009, 106(21):8507-8512.

64. Cardarelli L, Lam R, Tuite A, Baker LA, Sadowski PD, Radford DR, Rubinstein JL, Battaile KP, Chirgadze N, Maxwell KL, Davidson AR: The crystal structure of bacteriophage HK97 gp6: defining a large family of head-tail connector proteins. J Mol Biol 2010, 395(4):754-768.

65. Olia AS, Prevelige PE Jr, Johnson JE, Cingolani G: Three-dimensional structure of a viral genome-delivery portal vertex. Nat Struct Mol Biol 2011, 18(5):597-603.

66. Fokine A, Zhang Z, Kanamaru S, Bowman VD, Aksyuk AA, Arisaka F, Rao VB, Rossmann MG: The molecular architecture of the bacteriophage T4 neck. J Mol Biol 2013, 425(10):1731-44.

doi:10.1186/1471-2164-15-1027

Cite this article as: Lopes et al: Automated classification of tailed bacteriophages according to their neck organization. BMC Genomics 2014 15:1027.

\section{Submit your next manuscript to BioMed Central and take full advantage of:}

- Convenient online submission

- Thorough peer review

- No space constraints or color figure charges

- Immediate publication on acceptance

- Inclusion in PubMed, CAS, Scopus and Google Scholar

- Research which is freely available for redistribution 